Check for updates

Cite this: J. Mater. Chem. B, 2018, 6, 3703
Received 21st November 2017, Accepted 24th April 2018

DOI: $10.1039 / c 7 t b 03035 \mathrm{e}$

rsc.li/materials-b

\section{A hydroxamic acid-methacrylated collagen conjugate for the modulation of inflammation-related MMP upregulation}

\author{
He Liang, ${ }^{\text {ab }}$ Stephen J. Russell, ${ }^{a}$ David J. Wood ${ }^{b}$ and Giuseppe Tronci (D) *ab
}

\begin{abstract}
Medical devices with matrix metalloproteinase (MMP)-modulating functionality are highly desirable to restore tissue homeostasis in critical inflammation states, such as chronic wounds, rotator cuff tears and cancer. The introduction of MMP-modulating functionality in such devices is typically achieved via loading of either rapidly diffusing chelating factors, e.g. EDTA, or MMP-cleavable substrates, raising issues in terms of non-controllable pharmacokinetics and enzymatic degradability, respectively. Aiming to accomplish inherent, long-term, device-induced MMP regulation, this study investigated the synthesis of a hydroxamic acid (HA)-methacrylated collagen conjugate as the building block of a soluble factor-free MMPmodulating hydrogel network with controlled enzymatic degradability. This was realised via a two-step synthetic route: (i) type I collagen was functionalised with photonetwork-inducing methacrylic anhydride (MA) adducts in the presence of triethylamine (TEA); (ii) this methacrylated product was activated with a water-soluble carbodiimide prior to reaction with hydroxylamine, resulting in MMP-chelating HA functions. Nearly-quantitative methacrylation of collagen amines was observed via 2,4,6-trinitrobenzenesulfonic acid (TNBS) assay; this was key to avoiding intramolecular crosslinking side reactions during the carbodiimidemediated activation of collagen carboxyl groups. The molar content of HA adducts was indirectly quantified via the conversion of remaining carboxyl functions into ethylenediamine (EDA), so that $12-16$ mol\% HA was revealed in the conjugate by both TNBS and Ninhydrin assays. Resulting UV-cured, HA-bearing collagen hydrogels proved to induce up to $\sim 13$ and $\sim 32$ RFU\% activity reduction of MMP-9 and MMP-3, respectively, following 4-day incubation in vitro, whilst displaying an averaged mass loss in the range of $8-21 \mathrm{wt} \%$. Dichroic and electrophoretic patterns of native type I collagen could still be observed following the introduction of $\mathrm{HA}$ adducts, suggesting preserved triple helix architecture and chemical sequence in respective HA-methacrylated collagen conjugate. No hydrogel-induced toxic response was observed following the 4-day culture of G292 cells, whilst a lower compression modulus and gel content were measured in HA-bearing compared to methacrylated hydrogels, likely related to HA radical scavenging activity. The novel synthetic strategies described in this work provide a new insight into the systematic chemical manipulation of collagen materials aiming at the design of biomimetic, inflammation-responsive medical devices.
\end{abstract}

\section{Introduction}

Matrix metalloproteinases (MMPs) are a family of zinc- and calcium-dependent proteinases, which play a key role in breaking down extracellular matrix (ECM) proteins. The levels of MMPs are precisely regulated and are responsible for key physiological events such as homeostasis, tissue remodelling, wound healing and angiogenesis. ${ }^{1-7}$ Together with their beneficial role, numerous clinical studies have indicated that the overexpression of MMPs

${ }^{a}$ Clothworkers' Centre for Textile Materials Innovation for Healthcare, School of Design, University of Leeds, UK

${ }^{b}$ Biomaterials and Tissue Engineering Research Group, School of Dentistry, St. James's University Hospital, University of Leeds, UK. E-mail: g.tronci@leeds.ac.uk is associated with several inflammatory states, such as the ones found in chronic wounds, rotator cuff tears and cancer. ${ }^{8-13}$ For example, elevated levels of MMPs are found in synovial fluid samples from the glenohumeral joints of patients with massive rotator cuff tears. ${ }^{14,15}$ Likewise, 5 - to 10 -fold increases in MMP levels were found in chronic leg ulcers compared to acute healing wounds; these elevated levels of MMPs explain the turnover in chronic wounds and failed wound closure. ${ }^{16-19}$ Here, the imbalance between MMPs and tissue inhibitors of MMPs (TIMPs) promotes constant degradation of neo-tissues and region-specific growth factors, so that the typical healing process is impaired. In light of the critical role MMPs play in maintaining the integrity of the tissue ECM, growing research has therefore focused on the control of MMP 
expression aiming to manage inflammation and restore physiological homeostasis.

To date, 26 human MMPs have been found, which can be classified depending on the substrate specificity and molecular structure into at least four different classes, i.e. stromelysins (MMP-3, $-10,-11,-7$, and -12), collagenases (MMP-1, -8, -13, and -18), gelatinases (MMP-2 and -9) and membrane types MMPs (MMP-14, $-15,-16$, and -17)..$^{20}$ MMPs, and especially collagenases, cleave all three $\alpha$ chains of native type I, II and III collagens at a covalent bond between the glycine residue and the leucine or isoleucine residue, located approximately three-fourths of the way down the molecules from the N-terminus. ${ }^{21,22}$ Despite their classification, all MMPs present a catalytic zinc-binding domain, as well as a cysteine-containing pro-peptide domain at the molecular level. ${ }^{23}$ The cysteine residue is bound to the zinc atom when the enzyme is secreted in its latent conformation (pro-MMP). When the cysteine residue is dissociated from the zinc, the active site of the enzyme is exposed, triggering proteolytic activity. In vivo, the proteolytic removal of the pro-domain for MMP activation is mediated by other proteinases, including MMPs, whilst MMP regulation can be further achieved by complexation with TIMPs, which block access to the active site. ${ }^{24}$

Aiming to control inflammation-related MMP overexpression via systemic administration in vivo, the use of soluble factors has been widely investigated, whereby two main research approaches have been pursued:

(i) Synthesis of new TIMPs to restore the physiological MMP/ TIMP balance via systemic TIMP administration. However, despite several molecules having been synthesised and proposed for clinical use as TIMPs over the last 20 years, e.g. [4-( $N$-hydroxyamino)- $2 R$ isobutyl-3S-(thiopen-2-ylthiomethyl)-succinyl]-L-phenylalanine- $N$ methylamide (Batimastat ${ }^{\circledR}$ ) and $N$-[2,2-dimethyl-1-(methylcarbamoyl)propyl]-2-[hydroxy-(hydroxycarbamoyl)methyl]-4-methylpentanamide (Marimastat $^{\mathbb{R}}$ ), their clinical translation has been hampered by limited specificity and dose-limiting side effects (e.g. musculoskeletal syndrome) following systemic administration. ${ }^{25-27}$

(ii) Application of chelating compounds aiming to revert the MMP configuration to the native, deactivated state. Chelating agents, such as ethylenediaminetetraacetic acid (EDTA), have been proposed as zinc-chelating species to induce complexation with the exposed active site and to possess broad range MMP deactivation. Although this strategy has been successfully employed in some commercial wound dressings made from either hydrolysed collagen (Biostep ${ }^{\circledR}$ ), oxidized regenerated cellulose and hydrolysed collagen (Promogran ${ }^{\mathbb{R}}$ ), or a collagen-sodium alginate-carboxylmethylcellulose composite (ColActive ${ }^{\mathbb{R}}$ ), ${ }^{28,29}$ challenges with respect to the controlled release of the chelating agents and long-term device functionality have to date been only partially addressed.

To avoid unwanted side and temporal effects and to achieve long-lasting MMP regulation in situ, the design of multifunctional polymer architectures has received growing attention aiming at soluble factor-free, device-based therapies. ${ }^{30,31}$ Synthetic polymers, i.e. polyacrylates $^{32}$ and their respective hydroxamic acid-bearing derivatives, ${ }^{26}$ have been proposed as proof-of-concept anti-MMP substrates, due to the presence of ionically-charged and metalchelating groups, respectively, along the polymeric backbone.
The concept of synthetic polymer-induced MMP complexation and proteolytic regulation has been transferred to inherently MMP-cleavable biopolymers, e.g. collagen and gelatin, to provide resulting medical devices with an additional biomimetic interface. Here, the amino acidic collagen phase of the medical device can act as an alternative enzymatic substrate. According to this concept, the device-induced uptake of MMP-rich biological fluids triggers MMP-induced peptide bond cleavage. In this way, the MMP activity can be diverted to the collagen layer and away from the surrounding microenvironment, so that homeostasis can be restored. This concept has proved promising towards the development of device-based strategies for proteolytic activity regulation, ${ }^{33}$ although rapid MMP-induced biodegradation may lead to device form-instability, ${ }^{34}$ non-controllable volumetric swelling, and poor mechanical properties in vivo, impacting on clinical performance.

These challenges have recently been addressed by the development of photo-curable crosslinking chemistries leading to tunable water-stable collagen networks. Photoinduced crosslinking proved key to realise collagen-based biomimetic systems with bespoke elasticity $^{35}$ and controlled triple helix organisation, ${ }^{36}$ whereby material biocompatibility and wound healing capability have been largely demonstrated. ${ }^{37-39}$ UV-cured covalent networks made of functionalised collagen triple helices have also been presented with integrated MMP-chelating functions, whose swelling and compression properties can be controlled in a relatively independent fashion, via variation of e.g. the type and extent of collagen functionalisation. ${ }^{40,41}$ On the other hand, current synthetic routes can only target a small content of easily-accessible functional groups, i.e. primary amines, along the collagen backbone, resulting in restricted customisation of material functionalities, e.g. with regard to MMP-modulation capability. In light of their chemical reactivity and low molar content, primary amino terminations of collagen are mainly employed for the generation of covalent crosslinks, ${ }^{42}$ so that the introduction of MMP-modulating chemical functions in the resulting covalent networks is challenging to accomplish and control independently the network crosslink density. Furthermore, currently-available crosslinking strategies of collagen still raise concerns with regard to the occurrence of irreversible, non-controllable crosslinking side reactions. ${ }^{43}$ Consequently, flexible synthetic routes are still to be developed to fully realise multifunctional collagen systems with an independentlycustomisable format, properties and functions.

In this study, we aimed to explore this challenge by building a hydroxamic acid-methacrylated collagen conjugate as a biomimetic building block for the synthesis of UV-cured hydrogel networks with MMP-modulating capability and independentlyregulated crosslink density. Hydroxamic acid (HA) adducts were introduced via reaction with hydroxylamine, whereby the well-known chelating activity of HA was hypothesised to serve as means for MMP complexation and deactivation. Methacrylate functions were introduced via derivatisation of collagen amino groups to prompt the synthesis of a UV-cured crosslinked network in order to achieve enhanced enzymatic stability in the resulting materials. In addition to mediating the synthesis of the crosslinked network, the derivatisation of collagen with methacrylate functions 
was also exploited to protect the highly reactive, terminal amino groups of (hydroxy-)lysine residues to allow the selective modification of carboxylic functions avoiding either irreversible and noncontrollable side reactions or time-consuming de-/protection work-up.

Further to the quantification of the degree of collagen methacrylation via 2,4,6-trinitrobenzenesulfonic acid (TNBS), a new method is proposed to assess the HA-induced consumption of carboxylic groups via indirect carboxylic acid amination in the respective methacrylated and HA-conjugated collagen.

\section{Materials and methods}

\subsection{Materials}

Rat tails were provided by the School of Dentistry, University of Leeds, UK. Type I collagen was isolated in-house from the rat tail tendons upon acidic treatment. ${ }^{44}$ Methacrylic anhydride (MA), hydroxylamine hydrochloride (HA), triethylamine (TEA) and 2-mercaptoethanol (2-ME) were purchased from Sigma-Aldrich. $N$-(3-Dimethylaminopropyl)- $N$ '-ethylcarbodiimide hydrochloride (EDC), Ninhydrin and $N$-hydroxysuccinimide (NHS) were purchased from Alfa-Aesar. 2-Hydroxy-1-[4-(2-hydroxyethoxy)phenyl]-2-methylpropan-1-one (I2959) was purchased from Fluorochem Limited (Glossop, UK). All other chemicals were purchased from SigmaAldrich.

\subsection{Collagen methacrylation}

Methacrylated collagen was prepared as previously reported. ${ }^{40}$ Briefly, type I collagen from rat tails (CRT) was dissolved $(0.25 \mathrm{wt} \%)$ in $17.4 \mathrm{mM}$ acetic acid solution and stirred at $4{ }^{\circ} \mathrm{C}$ until complete dissolution was obtained. 0.1 $\mathrm{M} \mathrm{NaOH}$ solution was added to achieve a $\mathrm{pH}$ of 7.5. MA and TEA were added at a molar ratio of either 25 or 35 with respect to the molar content of primary amino functions $\left(\sim 3 \times 10^{-4} \mathrm{~mol} \mathrm{~g}^{-1}\right)$ available along the collagen backbone. After 24 hours the reaction mixture was precipitated in 10-volume excess of pure ethanol for 8 hours, recovered by centrifugation and air dried.

\subsection{Covalent coupling of HA adducts on to methacrylated collagen}

Methacrylated collagen (0.6 wt\%) was vigorously stirred in $0.01 \mathrm{M}$ calcium-free phosphate buffer solution (PBS) until complete dissolution. EDC and NHS were added to the obtained solution to activate carboxylic acid terminations of collagen $\left([\mathrm{EDC}] \cdot[\mathrm{COOH}]^{-1}=6 ;[\mathrm{EDC}]=[\mathrm{NHS}] ;[\mathrm{COOH}]=1 \mathrm{mmol} \mathrm{g}^{-1}\right) .{ }^{45,46}$ Following 60 min activation, an equimolar (to EDC) content of 2-ME was added to quench EDC activation. After $10 \mathrm{~min}$ reaction, either 2 or 4 molar excess (with respect to collagen carboxyl functions) of hydroxylamine chloride was added to induce HA covalent coupling. After 24 hour reaction, the mixture was precipitated in 10-volume excess of pure ethanol for 8 hours and recovered by centrifugation. This process was repeated twice and the collagen precipitate was air-dried.

\subsection{Grafting of collagen carboxyl groups with ethylene diamine}

Methacrylated and respective HA-conjugated collagens were aminated with ethylene diamine (EDA). This was carried out to indirectly quantify (via TNBS and Ninhydrin) the degree of HA-mediated collagen functionalisation by measuring the HA-induced consumption of collagen carboxylic groups. Either methacrylated or HA-conjugated collagen (0.6 wt $\%$ ) was vigorously stirred in $0.01 \mathrm{M}$ calcium-free PBS and $17.4 \mathrm{mM}$ acetic acid, respectively, until complete dissolution. EDC and NHS $\left([\mathrm{EDC}] \cdot[\mathrm{COOH}]^{-1}=6 \mathrm{mmol} \mathrm{g}^{-1}\right.$; $\left.[\mathrm{EDC}]=[\mathrm{NHS}]\right)$ were added, and each solution was stirred for one hour before adding an equimolar EDC content of 2-ME. After 10 minutes, 50 molar excess of EDA was added. This allowed the grafting of EDA onto activated carboxyl groups of either methacrylated or HA-conjugated collagen, resulting in the introduction of, and the conversion of carboxyl functions to, terminal amino groups. Following 24 hour reaction, reaction solutions were precipitated in 10-volume excess of pure ethanol for 8 hours and recovered by centrifugation. The retrieved products were re-dissolved in either PBS or $17.4 \mathrm{mM}$ acetic acid, and precipitated again prior to air drying.

\section{$2.5(2,4,6)$-Trinitrobenzenesulfonic acid (TNBS) and} Ninhydrin assays

The TNBS assay was used to directly measure derivatisation of amino groups and to indirectly measure derivatisation of carboxyl groups into either methacrylate, EDA or HA groups, and also respective collagen functionalisation. Briefly, $11 \mathrm{mg}$ of dry samples was mixed with $1 \mathrm{~mL}$ of $4 \mathrm{wt} \% \mathrm{NaHCO}_{3}$ (pH 8.5) and $1 \mathrm{~mL}$ of $0.5 \mathrm{wt} \%$ TNBS solution at $40{ }^{\circ} \mathrm{C}$ under mild shaking. After 4 hour reaction, $3 \mathrm{~mL}$ of $6 \mathrm{M}$ HCL solution was added and the overall reaction mixture was incubated at $60{ }^{\circ} \mathrm{C}$ for one hour to dissolve any sample residues. The solution was then cooled to room temperature, mixed with $5 \mathrm{~mL}$ of distilled water, and extracted three times with $15 \mathrm{~mL}$ of diethyl ether to remove non-reacted TNBS reagent. All samples were read against a blank (not containing samples) using an UV-vis spectrophotometer (Model 6305, Jenway) at $346 \mathrm{~nm}$, and the content of free amino groups and the degree of functionalisation $(F)$ were calculated according to eqn (1) and (2), respectively, as follows:

$$
\begin{gathered}
\frac{\mathrm{mol}(\text { Lys })}{\mathrm{g}(\text { collagen })}=\frac{2 \times \operatorname{Abs}(346 \mathrm{~nm}) \times 0.02}{1.46 \times 10^{4} \times b \times x} \\
F=100-\frac{\operatorname{mol}(\text { Lys })_{\text {funct. }}}{\operatorname{mol}(\text { Lys })_{\text {coll. }}} \times 100
\end{gathered}
$$

where Abs (346 nm) is the UV absorbance value recorded at $346 \mathrm{~nm}, 2$ is the dilution factor, 0.02 is the volume of the sample solution (in litres), $1.46 \times 10^{4}$ is the molar absorption coefficient for 2,4,6-trinitrophenyl lysine (in $\mathrm{M}^{-1} \mathrm{~cm}^{-1}$ ), $b$ is the cell path length $(1 \mathrm{~cm}), x$ is the dry sample weight, whilst mol(Lys) $)_{\text {coll. }}$ and mol(Lys) funct. represent the total molar content of free amino groups in native and functionalised collagen, 
respectively. (Lys) is hereby used to recognise that lysines make the highest contribution to the molar content of collagen free amino groups, although contributions from hydroxylysines and amino termini are also taken into account.

The Ninhydrin assay was further used to confirm the degree of derivatisation of the collagen amino groups previouslyobtained via TNBS. $11 \mathrm{mg}$ of the dry sample was mixed with $4 \mathrm{~mL}$ of distilled water and $1 \mathrm{~mL}$ of $8 \mathrm{wt} \%$ Ninhydrin solution in acetone. The mixture was then incubated at $100{ }^{\circ} \mathrm{C}$ for $15 \mathrm{~min}$. To terminate the reaction, the mixture was cooled on ice and $1 \mathrm{~mL}$ of $50 \%(\mathrm{w} / \mathrm{v})$ ethanol was added. The amount of free amino groups was determined by reading the absorbance at $570 \mathrm{~nm}$ against a blank sample (UV-vis, Model 6305, Jenway). A standard calibration curve was prepared by carrying out the above-mentioned assay with a known mass of collagen. Three replicates were used for both TNBS and Ninhydrin measurements. Data are presented as mean \pm SD.

\subsection{UV-induced hydrogel formation}

Methacrylated collagen and respective HA-conjugated products were dissolved (1.2 wt $\%$ ) in $0.01 \mathrm{M}$ calcium-free PBS containing 1 wt $\%$ 2-hydroxy-4'-(2-hydroxyethoxy)-2-methylpropiophenone (I2959) photoinitiator. The solution was centrifuged at $3000 \mathrm{rpm}$ to remove any air bubbles, followed by casting $\left(600 \mu \mathrm{L}\right.$ well $\left.{ }^{-1}\right)$ onto a 24-well plate (Corning Costar) and UV curing (Spectroline, $346 \mathrm{~nm}, 8 \mathrm{~mW} \mathrm{~cm}{ }^{-2}$ ) for $30 \mathrm{~min}$ at each side of the dish (light-sample distance: $5 \mathrm{~cm}$ ). The formed hydrogels were rinsed with distilled water, dehydrated via an ascending series of ethanol solutions and air dried.

\subsection{Quantification of gel content}

The gel content was measured to investigate the overall portion of the covalent hydrogel network insoluble in $17.4 \mathrm{mM}$ acetic acid solution. ${ }^{47}$ Dry collagen networks $\left(m_{\mathrm{d}}: 0.01-0.02 \mathrm{~g}\right)$ were individually incubated in $2 \mathrm{~mL}$ of $17.4 \mathrm{mM}$ acetic acid solution for 24 hours. The resulting samples were further air dried and weighed. The gel content $(G)$ was calculated by the following equation:

$$
G=\frac{m_{1}}{m_{\mathrm{d}}} \times 100
$$

where $m_{1}$ is the dry mass after incubation. Three replicates were used and the data are presented as mean $\pm \mathrm{SD}$.

\subsection{Compression tests}

Air-dried UV-cured samples were equilibrated in PBS via overnight incubation at room temperature. The resulting PBSequilibrated hydrogel discs $(\varnothing: 18 \mathrm{~mm} ; h$ : 5-6 mm) were compressed at room temperature at a compression rate of $3 \mathrm{~mm} \mathrm{m^{-1 }}$ (Instron ElectroPuls E3000). A $250 \mathrm{~N}$ load cell was operated up to complete sample compression. Stress-strain curves were recorded and the compression modulus was quantified as the slope of the plot linear region at $25-30 \%$ strain. Three replicates were employed for each collagen network composition. Data are presented as mean $\pm \mathrm{SD}$.

\subsection{MMP activity and enzymatic degradation study}

Full-length human pro-MMP-3 (PF063, Merck Millipore, UK) and pro-MMP-9 (PF038, Merck Millipore, UK) were activated in TCNB buffer containing $50 \mathrm{mM}$ Tris, $10 \mathrm{mM} \mathrm{CaCl}_{2}, 150 \mathrm{mM}$ $\mathrm{NaCl}$ and $0.05 \% \mathrm{Brij}-23(\mathrm{w} / \mathrm{v})(\mathrm{pH} 7.5)$, in the presence of $p$-aminophenyl mercuric acetate (AMPA) with a final concentration of $1 \mathrm{mM}$ at $37^{\circ} \mathrm{C}$ for 24 hours and 2 hours respectively, according to the manufacturer's guidance. Dry collagen networks of known weight $\left(m_{\mathrm{d}}, n=4\right)$ were individually placed in a 24-well plate containing activated solutions of either MMP-3 or MMP-9; the plate was then incubated at $37{ }^{\circ} \mathrm{C}$ on a benchtop orbital shaker for 4 days. The concentration of activated MMPs was $60 \mathrm{ng} \mathrm{mL}^{-1}$ and was selected according to the MMP concentration found in either torn rotator cuff or chronic wound fluid. ${ }^{8}$ Sample-free solution controls of either activated or non-activated MMPs were used. A standard commercial assay (Abcam, ab112146) was used to detect the MMP activity in the supernatants; additionally, the MMP-incubated samples were collected, dehydrated with increasing distilled water/ethanol series (0, 25, 50, 80 and 100 vol\% ethanol) and air-dried. The mass of the retrieved dried samples was measured $\left(m_{4}, n=4\right)$ and the relative mass $\left(\mu_{\text {rel }}\right)$ was calculated using the equation shown below:

$$
\mu_{\text {rel }}=\frac{m_{4}}{m_{\mathrm{d}}} \times 100
$$

\subsection{Circular dichroism}

The circular dichroism (CD) spectra of native and functionalised samples were acquired using a Chirascan CD spectrometer (Applied Photophysics Ltd) using $0.2 \mathrm{mg} \mathrm{mL}^{-1}$ solutions in $17.4 \mathrm{mM}$ acetic acid or $0.01 \mathrm{M}$ PBS solution. Sample solutions $(n=2)$ were collected in quartz cells of $1.0 \mathrm{~mm}$ path length, whereby CD spectra were obtained with $2.0 \mathrm{~nm}$ band width and $20 \mathrm{~nm} \mathrm{~min}^{-1}$ scanning speed. A spectrum of the $17.4 \mathrm{mM}$ acetic acid control solution was subtracted from each sample spectrum.

$$
\theta_{\mathrm{mrw}, \lambda}=\frac{\mathrm{MRW} \times \theta_{\lambda}}{10 \times d \times c}
$$

where $\theta_{\lambda}$ is the observed molar ellipticity (degrees) at wavelength $\lambda, d$ is the path length and $c$ is the concentration $\left(0.2 \mathrm{mg} \mathrm{mL}^{-1}\right)$. $\mathrm{MRW}$ is the mean residue weight and equals to $91 \mathrm{~g} \mathrm{~mol}^{-1}$ for amino acids. $^{48}$

\subsection{Sodium dodecyl sulphate-polyacrylamide gel electrophoresis (SDS-PAGE)}

The samples were dissolved (1 wt $\%$ ) in SDS sample buffer containing $160 \mathrm{mM}$ Tris-HCl (pH 6.8), 2\% SDS, 26\% glycerol, and $0.1 \%$ bromophenol blue. The sample solutions were heated for 60 seconds at $90{ }^{\circ} \mathrm{C}$. $30 \mu \mathrm{L}$ of each sample solution was added onto $4 \%$ stacking gel wells and separated on $15 \%$ resolving gels (200 V, $45 \mathrm{~min}$ ). Protein bands were visualized after $60 \mathrm{~min}$ staining (0.1 wt\% Coomassie Blue, $12.5 \mathrm{vol} \%$ trichloroacetic acid) and 60 min treatment in water under mild shaking. The molecular weight of the resulting bands was approximately determined by measuring the relative mobility of the standard protein molecular weight markers. 


\subsection{Cell culture}

Cells from the G292 cell line were cultured in Dulbecco's modified Eagle's medium (DMEM), supplemented with 10\% fetal bovine serum (FBS), 1\% glutamine, and $2.5 \mathrm{mg} \mathrm{mL}$ penicillin-streptomycin, in a humidified incubator at $37{ }^{\circ} \mathrm{C}$ and $5 \% \mathrm{CO}_{2}$. Cells were passaged every 3 days with $0.25 \%$ trypsin/ $0.02 \%$ EDTA. Freshly-synthesised, UV-cured hydrogel samples were incubated in a 70 vol\% ethanol solution under UV light. Retrieved samples were washed in PBS three times, prior to cell seeding. G292 cells $\left(8 \times 10^{3}\right.$ cells $\left.\mathrm{mL}^{-1}\right)$ were seeded on top of the hydrogel (following UV disinfection) and incubated at $37{ }^{\circ} \mathrm{C}$ for 4 days. After incubation, the hydrogels $(n=6)$ were washed with PBS $(\times 3)$ and transferred to a new 24-well plate before adding the dying agent of Calcein AM and Ethidium homodimer-1; the plate was then incubated for 20 minutes away from light. Finally, live and dead stained hydrogels were placed onto a glass slide for fluorescence microscopy imaging (Leica DMI6000 B). Control cells were cultured on tissue culture plastic (negative) and killed by $30 \mathrm{~min}$-incubation in $70 \mathrm{vol} \%$ methanol (positive). Other than live/dead staining, cell viability was assessed using Alamar Blue assay (ThermoFisher Scientific, UK) according to the manufacturer's guidance.

\subsection{Statistical analysis}

Statistical analysis was carried out using OriginPro 8.5.1. The significance of difference was determined by one-way ANOVA and the post hoc Tukey test. A $p$ value of less than 0.05 was considered to be significantly different. Data are presented as mean \pm SD.

\section{Results and discussion}

In the following, the design of a HA-methacrylated collagen conjugate is presented as a building block for a biomimetic medical device with integrated MMP-modulating capability. The medical device is realised at the molecular scale via the synthesis of a UV-cured hydrogel network of methacrylated collagen triple helices, in which the MMP-modulating capability is achieved via the introduction of HA adducts to carboxyl groups of methacrylated collagen.

The sample nomenclature used in this work is as follows: methacrylated and respective HA-conjugated collagen precursors are coded as 'MAXX' and 'HAYY', respectively, where 'MA' and 'HA' indicate the type of collagen adduct, whilst ' $\mathrm{XX}$ ' and 'YY' describe the molar ratios of the respective monomer in the functionalisation reaction. EDA-reacted samples were coded as either 'MAXXEDA' or 'HAYY-EDA', respectively. Collagen networks are coded as either 'MAXX') or 'HAYY*', where '*' identifies the chemicallycrosslinked state, whilst other labels have the same meaning as previously-mentioned.

\subsection{Synthesis of HA-methacrylated collagen conjugate}

The TEA-catalysed reaction of MA with collagen proceeds via an amine-initiated nucleophilic addition/elimination mechanism and was therefore carried out prior to coupling with HA. This reaction with MA was exploited for two purposes (Scheme 1): (i) to introduce photoactive functions on to free amino terminations of collagen, e.g. (hydroxy-)lysines and amino termini, responsible for the subsequent formation of a hydrogel network; ${ }^{36,40}$ (ii) to protect highly-reactive amino terminations prior to selective derivatisation of carboxyl into MMP-chelating functional groups, so that intra-crosslinking side reactions could be minimised.

The MA-mediated functionalisation of collagen was confirmed via TNBS and the content of MA adducts proved to be controlled depending on the MA/Lys molar ratio selected during the reaction (Table 1). The TNBS assay of MA-reacted collagen samples revealed nearly-quantitative and reproducible consumption of primary amino groups, so that an averaged degree of functionalisation higher than $80 \mathrm{~mol} \%$ was observed ( $\left.F_{\mathrm{MA}}: 82-100 \mathrm{~mol} \%\right)$. Similar reactions have been described in the literature, ${ }^{40,49,50}$ although resulted in lower $F_{\text {MA }}$ values, suggesting that identified experimental conditions were crucial for the above findings. Although an $F_{\mathrm{MA}}$ value of $100 \mathrm{~mol} \%$ was recorded in sample MA35, a longer solubilisation time was
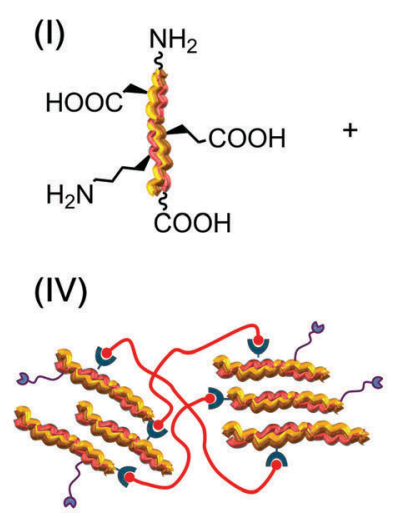<smiles>C=C(C)C(=O)OC(=O)C(=C)C</smiles>
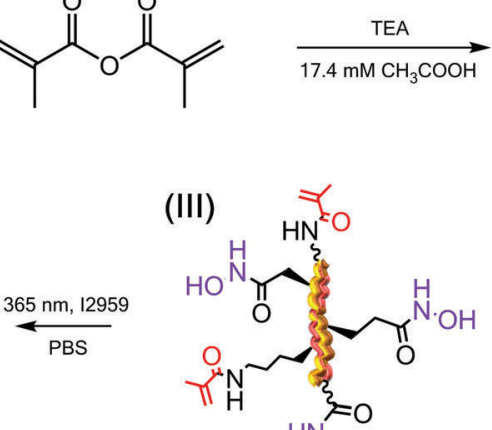

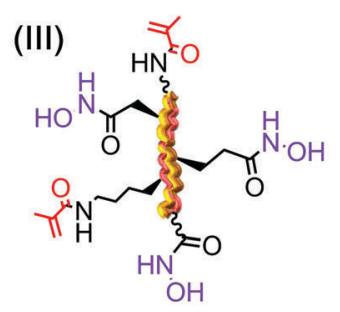

(II)
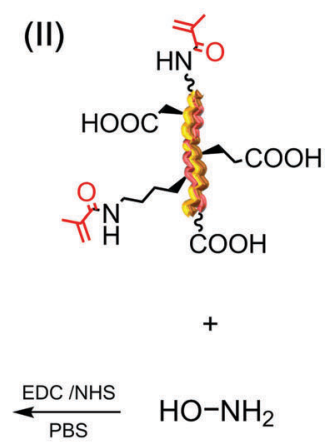

$\mathrm{HO}-\mathrm{NH}_{2}$

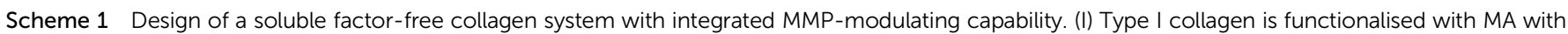

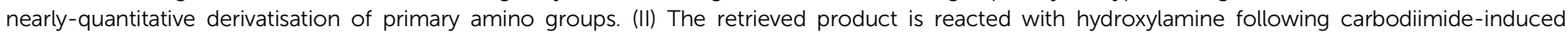

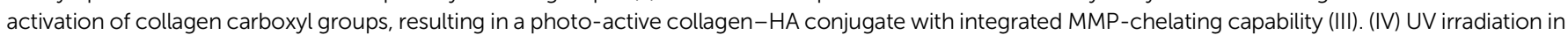

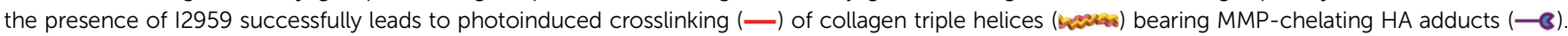


Table 1 Degree of methacrylation $\left(F_{M A}\right)$ determined via TNBS assay of collagen products $(n=3)$ obtained with varied MA/Lys molar ratio

\begin{tabular}{llc}
\hline Sample ID & {$[\mathrm{MA}][\mathrm{Lys}]^{-1}$} & $F_{\mathrm{MA}} / \mathrm{mol} \%$ \\
\hline MA10 & 10 & $82 \pm 2$ \\
MA25 & 25 & $94 \pm 1$ \\
MA35 & 35 & $100 \pm 0$
\end{tabular}

required with respect to sample MA25 ( $\left.F_{\mathrm{MA}}: 94 \mathrm{~mol} \%\right)$; consequently, the latter product was selected for future investigations.

To achieve the HA-methacrylated collagen conjugate, the carbodiimide-induced activation of sample MA25 was pursued in PBS, due to the limited solubility of collagen triple helices in organic solvents. The introduction of EDC and NHS in collagen solutions is known to induce the crosslinking reaction between collagen amino groups and NHS-activated collagen carboxyl groups, resulting in the formation of a gel. ${ }^{51}$ In agreement with previouslydiscussed TNBS results, solution gelation was not observed in our case, providing evidence that minimal unwanted intra-crosslinking reaction occurred between residual amino groups and activated carboxyl groups of collagen. At the same time, solubilisation of the retrieved, HA-reacted collagen product in an aqueous solution containing I2959 promptly led to the formation of a UV-cured hydrogel, as expected in light of the activation of covalentlycoupled MA functions.

\subsection{Quantification of HA-mediated functionalisation via an indirect amination strategy}

Following reaction with HA and confirmation of UV-induced gelation, the attention moved to the direct quantification of HA-mediated functionalisation in HA-methacrylated collagen samples. HA was covalently coupled to the carboxyl acids of collagen, so that no direct colorimetric assay, i.e. TNBS or Ninhydrin, could be carried out with the resulting product. Kenawy et al. reported on the functionalisation of poly( $N$-acryloxysuccinimide) with HA, whereby the resulting poly( $N$-hydroxyacrylamide) was successfully analysed via FTIR spectroscopy and ${ }^{1} \mathrm{H}-\mathrm{NMR} .{ }^{52}$ However, in the case of FTIR, the broad HA-related peaks at 3230 and $3480 \mathrm{~cm}^{-1}$ (due to $\mathrm{NH}$ and $\mathrm{OH}$ bonds) overlap with the ones related to the stretching vibrations of collagen $\mathrm{NH}$ bonds. Likewise, HA-related ${ }^{1} \mathrm{H}$-NMR signals at $\delta 3.0$ (singlet, $\mathrm{OH}$ ) and $\delta 4.10 \mathrm{ppm}$ (singlet, $\mathrm{NH}$ ) are likely to overlap with the ones of the main collagen amino acids, e.g. proline. Consequently, assessment of the HA content in HA-reacted collagen products can only be qualitative when using the above-mentioned methods.

In order to overcome this challenge, we aimed to indirectly quantify the molar content of HA by assessing the molar content of collagen carboxyl groups prior to and following reaction with HA, since the carboxyl groups in collagen were directly involved in the coupling reaction. To reach this goal, derivatisation of collagen carboxyl groups into TNBS- and Ninhydrin-detectable terminal amino groups was pursued in both methacrylated and HA-reacted collagen products via reaction with EDA (Scheme 2).

To avoid unwanted crosslinking reaction and promote selective grafting of EDA molecules, samples MA25, HA2 and HA4 were reacted with a 50-molar excess of EDA following carboxyl function activation with EDC/NHS. No gel formation was observed during the EDC-mediated activation of collagen following the reaction of EDC-activated collagen with EDA. These observations provided evidence that an EDA-grafted rather than a crosslinked product was obtained, supporting the validity of the proposed approach in selectively derivitising carboxyl groups into terminal amines. Table 2 shows values related to the molar content of EDA- and HA-related adducts, as

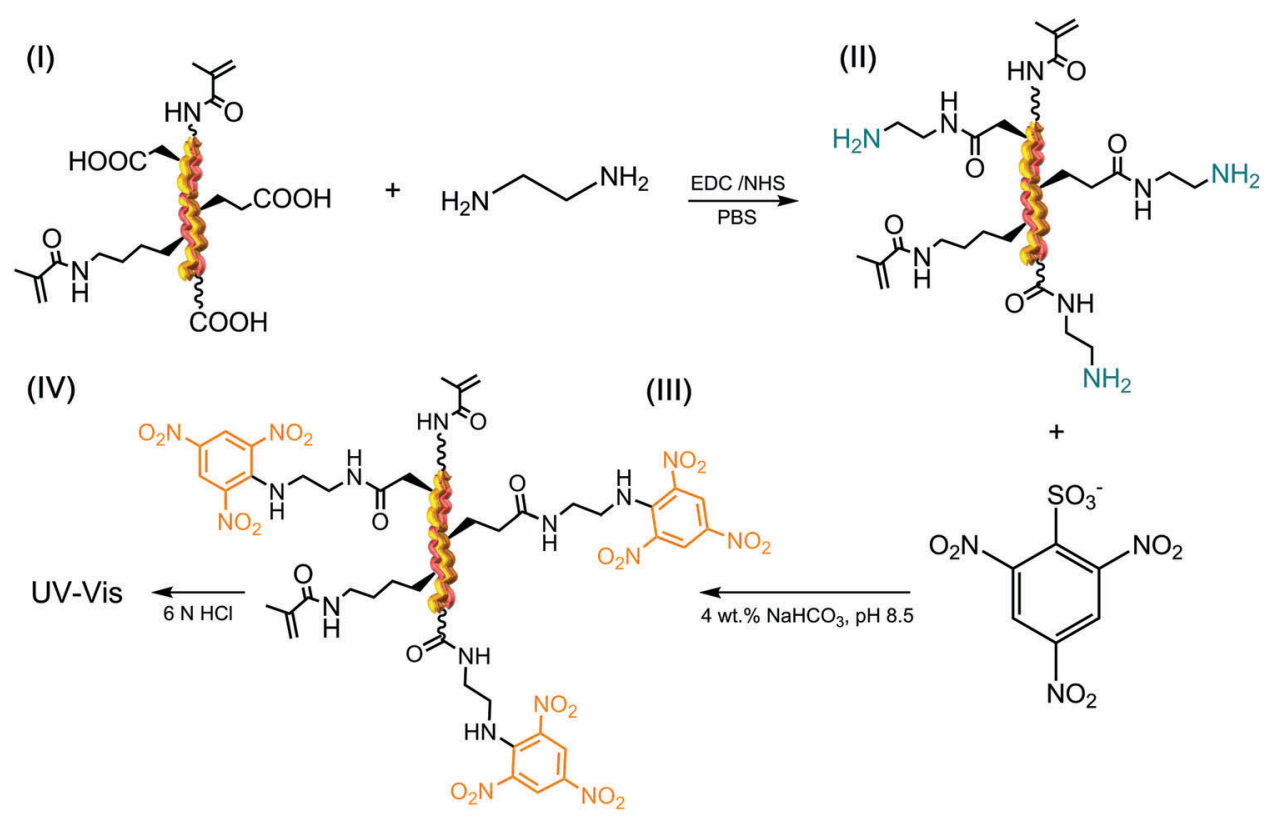

Scheme 2 Quantification of carboxyl terminations of collagen via an indirect amination strategy. Methacrylated collagen (I) was carbodiimide-activated and grafted with EDA, resulting in the direct conversion of carboxyl to primary amino functions (II). The aminated product was assessed with TNBS (III), allowing the indirect quantification of pristine carboxyl functions via UV-vis spectroscopy (IV). 
Table 2 Degree of HA-mediated functionalisation $\left(F_{\mathrm{HA}}\right)$ determined in EDA-reacted, methacrylated, and respective HA-conjugated samples $(n=3)$ via both TNBS and Ninhydrin assays. EDA terminal amino groups were used to indirectly quantify the consumption of carboxyl groups and consequent coupling of HA adducts. n.a.: not applicable

\begin{tabular}{|c|c|c|c|c|c|c|}
\hline \multirow[b]{2}{*}{ Sample ID } & \multicolumn{2}{|c|}{$[\mathrm{EDA}] / \mathrm{mol} \mathrm{g}^{-1}\left(\times 10^{-4}\right)$} & \multicolumn{2}{|c|}{$[\mathrm{HA}] / \mathrm{mol} \mathrm{g}^{-1}\left(\times 10^{-4}\right)$} & \multicolumn{2}{|l|}{$F_{\mathrm{HA}}^{a} / \mathrm{mol} \%$} \\
\hline & TNBS & Ninhydrin & TNBS & Ninhydrin & TNBS & Ninhydrin \\
\hline MA25-EDA & $1.61 \pm 0.08$ & $1.48 \pm 0.05$ & n.a. & n.a. & n.a. & n.a. \\
\hline HA2-EDA & $0.14 \pm 0.04$ & $0.17 \pm 0.03$ & $1.47 \pm 0.04$ & $1.21 \pm 0.08$ & $14.7 \pm 0.4$ & $12.1 \pm 0.7$ \\
\hline HA4-EDA & $0.06 \pm 0.02$ & $0.09 \pm 0.04$ & $1.55 \pm 0.06$ & $1.37 \pm 0.09$ & $15.5 \pm 0.6$ & $13.7 \pm 0.9$ \\
\hline
\end{tabular}

well as the respective degrees of HA-mediated collagen functionalisation $\left(F_{\mathrm{HA}}\right)$.

EDA-induced grafting was confirmed by the fact that an increased content of free amino groups was recorded in sample MA25-EDA ([R-NH $]$ ]: $1.80 \times 10^{-4} \mathrm{~mol} \mathrm{~g}^{-1}$ ) with respect to the one measured in sample MA25 ([Lys]: $0.19 \times 10^{-4} \mathrm{mmol} \mathrm{g}^{-1}$ ), so that an overall grafted EDA content of $1.61 \times 10^{-4} \mathrm{~mol} \mathrm{~g}^{-1}$ was successfully achieved. Most importantly, the molar content of amino groups was found to be lower in both HA-conjugated products, i.e. samples HA2-EDA ([EDA]: $0.14 \times 10^{-4} \mathrm{~mol} \mathrm{~g}^{-1}$ ) and HA4-EDA ([EDA]: $0.06 \times 10^{-4} \mathrm{~mol} \mathrm{~g}^{-1}$ ).

These results confirm the successful coupling of HA adducts on to collagen carboxyl functions. Considering an overall amount of carboxyl groups of $1 \times 10^{-3} \mathrm{~mol} \mathrm{~g}^{-1}$ in rat tail collagen, ${ }^{43,44}$ the above-mentioned results corresponded to an averaged degree of HA-mediated functionalisation of up to $\sim 16$ mol\% (Table 2). Together with TNBS, Ninhydrin assay was also carried out to further confirm the above findings. Also in this case, a decreased molar content of amino groups was recorded in HA-conjugated compared to methacrylated collagen samples. An averaged $F_{\mathrm{HA}}$ value of up to $\sim 14 \mathrm{~mol} \%$ was obtained, well in line with previous TNBS data.

Results obtained via collagen amination and colorimetric assays therefore provided supporting evidence that covalent coupling of both methacrylate and HA adducts could be selectively accomplished by independently targeting collagen amino and carboxyl groups, respectively. The nearly-quantitative lysine functionalisation with MA adducts proved key to selectively target free carboxyl functions of methacrylated collagen in a controlled fashion. The presented synthetic approach therefore proved reliable to enable the quantification of free carboxyl groups avoiding either the occurrence of well-reported, undesired intramolecular crosslinking reactions between collagen amino and carboxyl functions, or the employment of time-consuming de-/protection work-up. ${ }^{53,55-57}$ Although low variation in HA coupling was measured by both TNBS and Ninhydrin assays, a significant effect on the MMP-modulating capability of the resulting UV-cured hydrogels was observed (Section 3.4). It is expected that a wider range of functionalisation can be obtained by further adjusting the molar ratio of hydroxylamine with respect to carboxylic acid groups of methacrylated collagen.

\subsection{Analysis of collagen conformation}

SDS-PAGE was used to both elucidate the chemical composition of reacted products and explore the triple helix organisation of methacrylated and HA-conjugated products. Retention of the triple helix architecture of collagen is key to enable the resulting materials with chemotactic functionality, enhanced mechanical properties and decreased swellability. ${ }^{39}$ Although we have previously confirmed that collagen methacrylation had a minimal effect on the collagen triple helix structure, ${ }^{40}$ the reaction of collagen-based polypetides with hydroxylamine has been reported to catalyse the chemical cleavage of peptide bonds between asparagine and glycine residues ${ }^{58-60}$ which is likely to induce denaturation of collagen triple helices. Despite the low (up to $9 \mathrm{mM}$ ) hydroxylamine concentrations employed in this study, it was important to confirm that no degradation product was formed in the reacted species, aiming not to compromise the chemical sequence and organisation of native collagen.

In-house extracted type I rat tail collagen was confirmed to display electrophoretic bands of monomeric $\alpha$-chains ( $\sim 100 \mathrm{kDa})$ and dimeric $\beta$-components ( $\sim 200 \mathrm{kDa})$, as shown in Fig. 1(A). Each of these bands was observed in the electrophoretic patterns of samples MA25, HA2 and HA4, whilst no further band could be identified. SDS-PAGE data therefore supported the fact that no detectable collagen degradation occurred during the reaction with hydroxylamine. The minimal pattern variation in the electrophoretic reference bands associated with reacted, with respect to, native collagen also suggested no alteration in the native triple helix organisation following collagen functionalisation.

To further elucidate this point, CD spectroscopy was carried out. Type I collagen presents a unique CD spectrum with a negative peak at $197 \mathrm{~nm}$ and a positive peak at $221 \mathrm{~nm} ;{ }^{36,61,62}$ these characteristic peaks could be identified in all collagen samples (Fig. 1B), confirming the presence of polyproline-II and triple helices, respectively. ${ }^{63}$ The magnitude ratio of positive to negative peak (RPN) in the CD spectra provides an indication of the content of collagen triple helices. ${ }^{40}$ The RPN values measured in the CD spectra of samples MA25, HA2 and HA4 were $0.113,0.094$ and 0.101 , respectively, which were only slightly lower than the RPN value measured in the CD spectra of native collagen (RPN: 0.122). By normalising the RPN value measured from functionalised samples with respect to the one of native collagen, a degree of triple helix preservation of at least 77 RPN\% is observed. These results provide supporting evidence that collagen triple helices could still be preserved in HA-conjugated collagen samples, although at a lower extent with respect to the case of methacrylated samples.

The preservation of collagen triple helices in HA-conjugated collagen is an interesting finding, given that additional HA, 

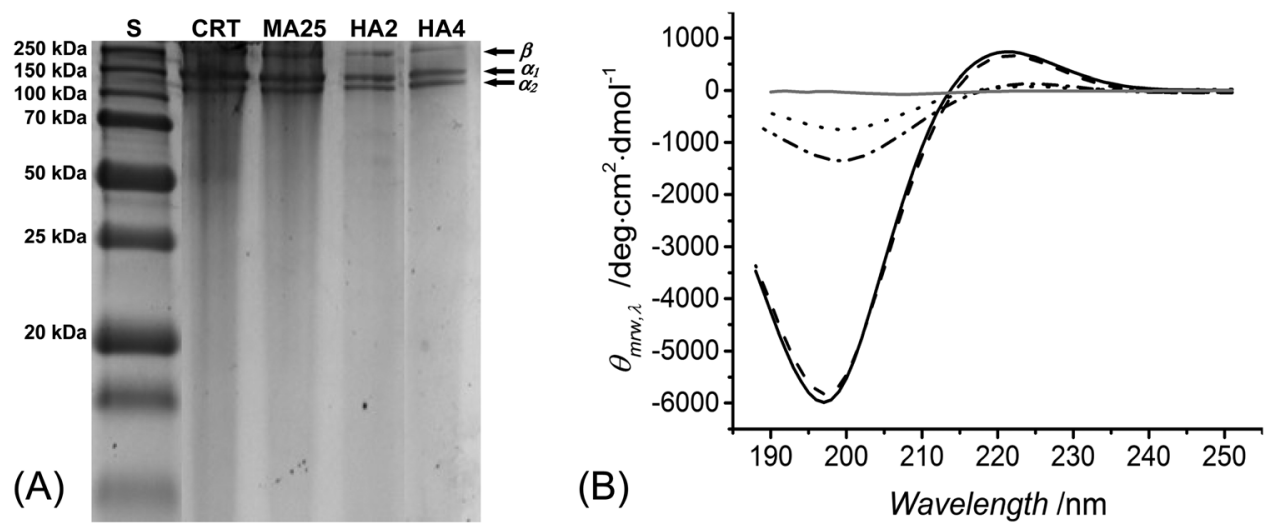

Fig. 1 (A) SDS-PAGE analysis of standard (S), in-house isolated type I collagen from rat tail (CRT), as well as samples of MA25, HA2 and HA4. (B) Far-UV CD spectra $(n=2)$ of samples of collagen (black solid line, RPN $\sim 0.122)$, MA25 (black dash line, RPN $\sim 0.113$ ), HA2 (black dash and dot, RPN $\sim 0.094$ ), HA4 (black dot, RPN 0.101) and gelatin (grey).

besides MA, adducts are covalently coupled to the collagen backbone. This finding may be explained by the fact that the overall HA molar content was limited up to only $16 \mathrm{~mol} \%$ of collagen carboxyl functions, suggesting that increasing the degree of HA-mediated functionalisation may negatively affect the triple helix organisation of native collagen.

\subsection{Hydrogel's impact on MMP activities and related enzymatic degradability}

Following elucidation of the molecular architecture, protein organisation, and UV-cured network formation, hydrogel proteolytic degradability and MMP-regulation capability were studied in vitro with either MMP-3 or MMP-9. Both MMPs were selected in light of their documented overexpression in inflammation states, such as in the case of rotator cuff tears, ${ }^{5}$ chronic wounds $^{3,9}$ and osteoarthritis. ${ }^{64}$ Fig. 2(A) shows the MMP-3 activity of aqueous media following 4-day conditioning with samples MA25*, HA2* and HA4*, with respect to sample-free MMP-3 solutions.

All samples proved to induce a significant reduction in MMP-3 activity $(p<0.001)$, with samples HA2* (MMP-3: $81 \pm$ 4 RFU\%) and HA4 (MMP-3: $70 \pm 6$ RFU\%) displaying significantly higher effect $(p<0.01)$ with respect to methacrylated hydrogels (MMP-3: $87 \pm 2$ RFU\%). Together with the analysis on supernatants, the relative mass of 4-day incubated samples was measured and compared to that of a control sample of native, non-crosslinked CRT (Fig. 2B). Hydrogel HA4* proved to show the highest relative mass $\left(\mu_{\text {rel }}: 96 \pm 2 \mathrm{wt} \%\right)$, which was significantly higher than the ones recorded in samples HA2* $\left(\mu_{\text {rel }}: 79 \pm 4 \mathrm{wt} \%\right)$ and MA25* $\left(\mu_{\text {rel }}: 79 \pm 3 \mathrm{wt} \%\right)$. As expected, the lowest relative mass was found in the control sample $\left(\mu_{\text {rel }}\right.$ : $44 \pm 4 \mathrm{wt} \%$ ), due to the absence of a crosslinked network at the molecular scale.

Similar trends were confirmed following sample incubation with MMP-9-containing aqueous media (Fig. 3A). MMP-9 activities were found to be reduced to either $88 \pm 5$ or $87 \pm 4$ RFU\% following supernatant conditioning with either sample HA2* or HA $4^{*}$, with significant difference observed in comparison to the supernatant treated with sample MA25* (MMP-9: $98 \pm 1$ RFU\%). As observed with MMP-3, sample CRT exhibits the lowest relative mass ( $\mu_{\text {rel }}: 57 \pm 7 \mathrm{wt} \%$ ) (Fig. 3B), whilst both MMP-chelating hydrogels displayed more than $90 \mathrm{wt} \%$ averaged relative mass. In comparison, sample MA25* displayed a lower relative mass ( $\left.\mu_{\text {rel }}: 87 \pm 3 \mathrm{wt} \%\right)$, which proved significantly different with respect to the ones of all crosslinked samples.

MMP-3 and MMP-9 belong to two different classes of MMPs, namely stromelysins and gelatinases, respectively. Accordingly,
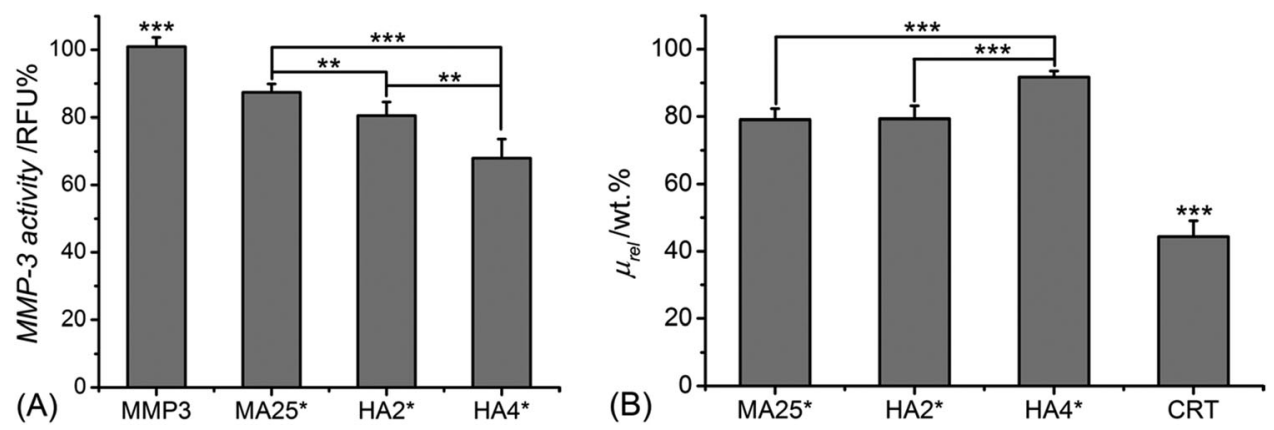

Fig. 2 (A) MMP-3 activity measured in vitro following 4-day incubation with either no sample (MMP-3) or samples of MA25*, HA2* and HA4*. Results are normalised to the MMP-3 activity recorded in a sample-free medium. (B) Relative mass of samples of CRT, MA25*, HA2* and HA4* following 4-day incubation in MMP-3-containing aqueous solution (60 $\left.\mathrm{ng} \mathrm{mL}^{-1} \mathrm{MMP}-3\right) .{ }^{*} p<0.05,{ }^{*} p<0.01$ and ${ }^{* \star *} p<0.001$ are considered significant $(n=4)$. 

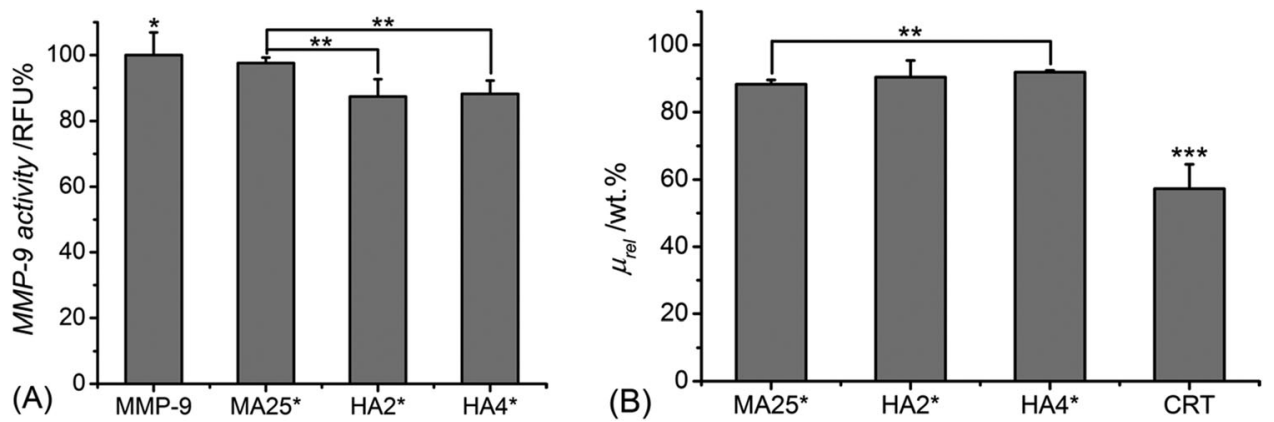

Fig. 3 (A) MMP-9 activity measured in vitro following 4-day incubation with either no sample (MMP-9) or samples of MA25*, HA2* and HA4*. Results are normalised to the MMP-9 activity recorded in a sample-free medium. (B) Relative mass of samples of CRT, MA25*, HA2* and HA4*, following 4-day incubation in MMP-9-containing aqueous solution (60 $\left.\mathrm{ng} \mathrm{mL}^{-1} \mathrm{MMP}-9\right) .{ }^{*} p<0.05,{ }^{* *} p<0.01$ and ${ }^{* * *} p<0.001$ are considered significant $(n=4)$.

MMP-3 presents broad substrate specificity and induces the activation of collagenases, e.g. MMP-1, a potent collagendegrading enzyme. In contrast, MMP-9 is reported to preferentially degrade denatured collagen, i.e. gelatin, rather than collagen triple helices. ${ }^{65}$ Our gravimetric data support the broader substrate specificity presented by MMP-3, whereby a lower relative mass was recorded in both native collagen and hydrogel samples following incubation with MMP-3, compared to the case of the MMP-9-incubated samples. The broad specificity of MMP-3 compared to MMP-9 was also reflected by the higher hydrogel-induced activity reduction of the former compared to the latter enzyme. In light of MMP-9 specificity towards single rather than triple helices of collagen, the above-mentioned observations provide further indirect evidence of triple helix retention in both methacrylated and HA-conjugated collagen hydrogels. The non-preferential specificity of MMP-9 towards collagen triple helices was also in agreement with the insignificantly different MMP-9 activity (Fig. 3A) and relative sample mass (Fig. 3B) recorded following 4-day incubation with either sample HA4* or sample HA2* despite the increased HA content measured in the former compared to the latter system (Table 2). This observation correlated with previously-reported CD spectra (Fig. 1B), which indicated an increased triple helix-related RPN value in the spectra of sample HA4 (RPN: 0.101), with respect to the case of sample HA2 (RPN: 0.094). Other than MMP-9, the fact that the obtained hydrogel samples promoted increased deactivation of MMP-3 may be beneficial to control the excessive activation of tissue-detrimental collagenases. ${ }^{24}$ Overall, the above-mentioned proteolytic and gravimetric results with both MMP-3 and MMP-9 demonstrated the MMP-modulating capability induced by the functionalisation of the collagen backbone with MMP-chelating HA adducts. In contrast to endogenous MMP inhibitors, the HA-conjugated collagen hydrogels obtained in this study induce proteolytic modulation by binding to the active $\mathrm{Zn}$ site and a non-active $\mathrm{Ca}$ site inducing the MMP to adapt its native conformation.

Until now, there have been limited studies on targeting the overexpression of MMPs due to the undesired side effects associated with the administration of soluble MMP inhibitors. Although HA-conjugated synthetic polymer systems have been presented in the past, no collagen variant has yet been developed, likely related to the challenges associated with the control and manipulation of the collagen architecture and the occurrence of crosslinking side reactions during collagen modification. Skarja et al. reported HA-containing microspheres prepared via derivatisation of poly(methyl methacrylate-co-methacrylic acid) and respective microsphere-induced reduction of the activity of MMP-2, $-3,-8$ and $-13 .{ }^{26}$ Other studies investigated the effect of ovine-based collagen dressing on a broad spectrum of MMP reduction but led to undesired clinical outcomes. ${ }^{66-69} \mathrm{~A}$ layer by layer SiRNA coated nylon bandage was reported to yield rapid chronic wound closure in a diabetic mice model silencing $60 \%$ of MMP-9 activity in a two week study; ${ }^{70}$ however, the biocompatibility of nylon may need to be considered further to enable the clinical applicability of the resulting device.

We have recently reported a $4 \mathrm{VBC}$-functionalised collagen hydrogel with covalently-bound electron rich aromatic adducts, such that the MMP-9 activity was reduced by $50 \%$ in 4 days in vitro. ${ }^{38}$ With respect to that study, the hereby presented HAconjugated formulation is likely to offer additional advantages, given the possibility to control the MMP-modulating capability independently of the crosslink density of the network.

\subsection{Characterisation of physical properties}

Once the MMP-modulating functionality of HA-bearing hydrogels was addressed, the attention moved to the physical characterisation of the hydrogels. The gel content was quantified gravimetrically to gain information on the crosslink density and the overall portion of the non-extractable crosslinked material (Fig. 4A). Methacrylated hydrogels MA25* revealed the highest gel content of over $90 \mathrm{wt} \%$ ( $G: 93 \pm 1 \mathrm{wt} \%$ ), which was higher than the one measured with samples HA2* ( $G: 87 \pm 1 \mathrm{wt} \%)$ and $\mathrm{HA}^{*}$ ( $\left.G: 85 \pm 5 \mathrm{wt} \%\right)$.

Interestingly, despite a lower gel content in HA-based compared to methacrylated networks, samples $\mathrm{HA}^{*}$ could still display decreased mass loss and induce increased reduction of MMP activity, further confirming the effect of covalentlycoupled HA adducts on to the collagen backbone. In line with the gel content data, the compression modulus (Fig. 4B) proved to be significantly affected by the hydrogel formulation, so that sample MA25* showed significantly higher compressive modulus $\left(E_{\mathrm{c}}: 128 \pm 17 \mathrm{kPa}\right)$ with respect to samples HA2* $\left(E_{\mathrm{c}}: 81 \pm 10 \mathrm{~Pa}\right)$ and $\mathrm{HA}^{*}\left(E_{\mathrm{c}}: 72 \pm 16 \mathrm{kPa}\right)$. 

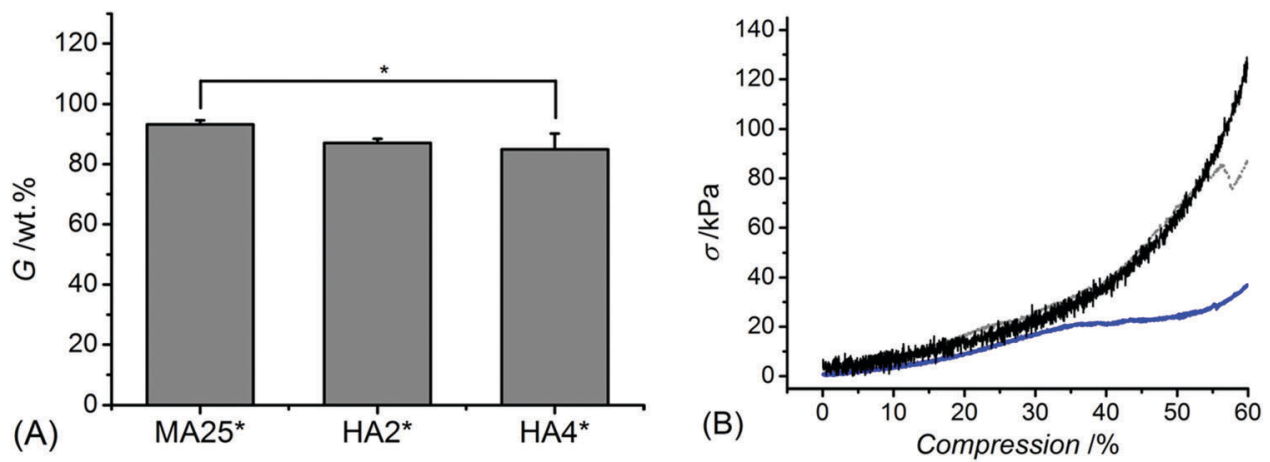

Fig. 4 Gel content (G, (A)) and representative wet-state stress-compression curves (B) of samples MA25* (black), HA2* (grey) and HA4* (blue). ${ }^{*} p<0.05$ is considered significant $(n=3)$.

HA derivatives are known to display radical scavenging functionality, ${ }^{71}$ suggesting that the presence of HA adducts in the methacrylated collagen precursor impacts on the half-life of UVgenerated radicals, crosslinking reaction kinetics and network crosslink density. This hypothesis is likely to explain the trends in the gel content across the different formulations, since a decreased gel content and compression modulus were observed in HA-bearing compared to methacrylated samples. The indirectly-observed radical scavenging functionality of HA-bearing hydrogels could also play a major role in controlling the excessive upregulation of the reactive oxygen species found in e.g. chronic wounds, providing an additional capability in controlling inflammation.

\subsection{In vitro cell cytotoxicity}

The potential use of the HA-conjugated collagen hydrogels in a biological system was evaluated by investigating the cellular response of osteosarcoma cells when cultured in direct contact with the material. Cell metabolism and viability were measured by Alamar blue assay at day 4 and 7 of cell culture (Fig. 5), and exemplarily confirmed via live/dead staining on cells seeded on HA-conjugated collagen network HA4* following 4-day culture (Fig. 6).

At day 4, the highest and lowest cell viability values were recorded (via Alamar blue assay) in cells cultured onto either tissue culture plastic (TCP) or native collagen, whilst intermediate

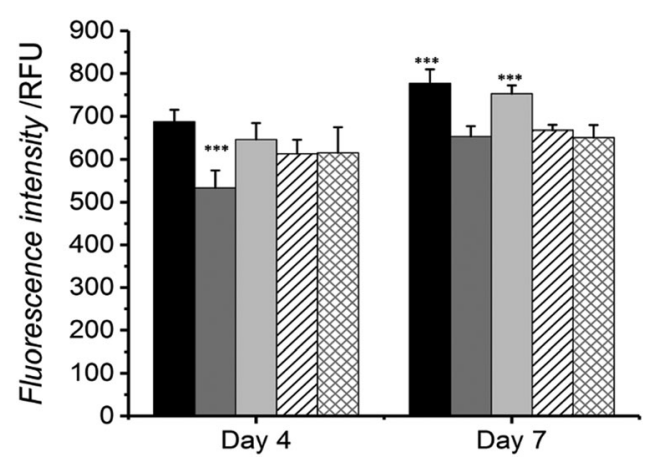

Fig. 5 Alamar blue assay carried out with G292 osteosarcoma cells over 7 days. Cells were cultured onto either tissue culture plastic (black) and native collagen (grey) controls or samples of MA25* (light grey), HA2* (black, sparse patterned) and $\mathrm{HA}^{*}$ (grey, sparse patterned). ${ }^{* *}$ indicates significantly-different means with respect to other means at each specific time point $(p<0.001, n=6)$.
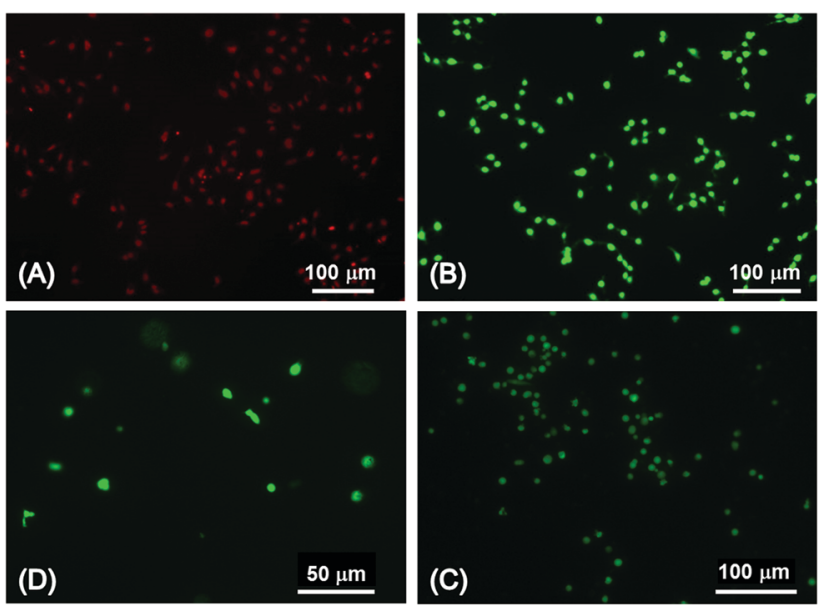

Fig. 6 Typical live/dead staining of G292 osteosarcoma cells following 4-day culture. (A) and (B) show the positive and negative controls at day 4 of cell culture, respectively. (C) and (D) describe the live/dead staining of G292 cells following 4-day seeding on hydrogel $\mathrm{HA}^{*}$ at the magnification of $\times 10$ and $\times 20$, respectively.

viability levels were seen in cells cultured with samples MA25*, $\mathrm{HA}^{*}$ and HA4* (Fig. 5). No significant difference in cell viability was observed among the three UV-cured sample groups compared to the TCP group, whilst all of them were found to display increased cellular tolerance with respect to the sample of native collagen. At day 7 , the highest cellular viability was measured via Alamar blue assay on cells cultured onto both TCP control and MA25* sample, with no statistical difference between them. Similar trends were also recorded in cells cultured onto native collagen control as well as onto samples of $\mathrm{HA}_{2}^{*}$ and HA4*, although respective cell viability proved to be significantly lower than the one recorded in TCP and MA25* groups.

Interestingly, sample MA25* seemed to induce a similar level of cellular proliferation and metabolism in comparison to the TCP control and in contrast to HA-conjugated collagen networks; this result may be due to the increased mechanical strength of hydrogel MA25* with respect to hydrogels HA2* and HA4* Overall, these cell culture results successfully proved the high tolerability of all hydrogels tested in this study with G292 osteosarcoma cells. 
In agreement with the results obtained via Alamar blue assay, no hydrogel-induced toxic response was confirmed via live/dead staining of G292 cells following 4-day culture onto sample HA4*. In contrast to the positive control confirming the presence of dead (red) cells (Fig. 6A), only vital (green) cells were indeed detected in samples HA4* (Fig. 6C and D), as in the case of the negative control (Fig. 6B).

Many reports suggested that soluble HA derivatives exhibit cytotoxicity against several cell lines such as human fibroblast, leukaemia and cancer cells. ${ }^{72-75}$ This finding was not observed in this study as a high cell viability was observed following 7 day culture onto HA-bearing collagen hydrogels with respect to native collagen controls. This observation further supports the use of HA-coupled rather than HA-loaded systems for the prolonged and localised regulation of upregulated MMPs.

\subsection{Conclusion}

The synthesis of a HA-methacrylated collagen conjugate was successfully demonstrated for the development of soluble factor-free hydrogel photonetworks with integrated MMP-regulating capability. Sequential covalent coupling of methacrylate and HA adducts to collagen was selectively accomplished by independently targeting amino and carboxyl groups, respectively. In this way, the hydrogel MMP-regulating capability could be controlled independently of the collagen network crosslink density. Amination of carboxyl groups via grafting reaction with EDA proved successful to indirectly quantify the degree of HA-mediated collagen functionalisation by both TNBS and Ninhydrin assays. The resulting HA-conjugated collagen samples maintained their unique triple helical structure, although at a lower extent with respect to methacrylated samples. The hydrogel-induced reduction in activities of both MMP-3 and MMP-9 demonstrated the MMP-modulating capability induced by the covalent functionalisation of the collagen backbone with MMP-chelating HA adducts, whereby the inherent MMP substrate specificity was observed to play a role. Increased enzymatic stability and decreased compressive modulus were measured in HA-conjugated compared to methacrylated hydrogel networks, in agreement with the presence of covalently-coupled HA adducts. Such materials were also shown to be cell friendly when cultured with G292 osteosarcoma cells. The versatility of this HA-methacrylated collagen conjugate has great potential in bypassing the wellknown clinical problems associated with chelating agent-loaded medical products and provides a novel solution for the devicebased modulation of MMP overexpression in a variety of diseases.

\section{Conflicts of interest}

There are no conflicts to declare.

\section{Acknowledgements}

The authors gratefully acknowledge financial support provided by the EPSRC Centre for Innovative Manufacturing in Medical Devices (MeDe Innovation), as well as the Clothworkers' Centre for Textile Materials Innovation for Healthcare (CСTMIH).
Jackie Hudson and Sarah Myers are gratefully acknowledged for technical assistance with fluorescence microscopy as well as cell culture facilities.

\section{References}

1 R. J. Tan and Y. Liu, Matrix metalloproteinases in kidney homeostasis and diseases, Am. J. Physiol.: Renal, Fluid Electrolyte Physiol., 2012, 302(11), F1351-F1361.

2 J. Gaffney, et al., Multilevel regulation of matrix metalloproteinases in tissue homeostasis indicates their molecular specificity in vivo, Matrix Biol., 2015, 44, 191-199.

3 M. P. Caley, V. L. C. Martins and E. A. O'Toole, Metalloproteinases and Wound Healing, Adv. Wound Care, 2015, 4(4), 225-234.

4 D. G. Armstrong and E. B. Jude, The role of matrix metalloproteinases in wound healing, J. Am. Podiatric Med. Assoc., 2002, 92(1), 12-18.

$5 \mathrm{~J}$. Jacob, et al., Matrix metalloproteinase levels as a marker for rotator cuff tears, Orthopedics, 2012, 35(4), e474-e478.

6 S. De Giorgi, M. Saracino and A. Castagna, Degenerative disease in rotator cuff tears: what are the biochemical and histological changes?, Joints, 2014, 2(1), 26-28.

7 C. Gialeli, A. D. Theocharis and N. K. Karamanos, Roles of matrix metalloproteinases in cancer progression and their pharmacological targeting, FEBS J., 2011, 278(1), 16-27.

$8 \mathrm{~J}$. Jacob, et al., Matrix Metalloproteinase Levels as a Marker for Rotator Cuff Tears, Orthopedics, 2012, 35, 474-478.

9 L. Ravanti and V. M. Kahari, Matrix metalloproteinases in wound repair (review), Int. J. Mol. Med., 2000, 6(4), 391-407.

10 T. Osawa, T. Shinozaki and K. Takagishi, Multivariate analysis of biochemical markers in synovial fluid from the shoulder joint for diagnosis of rotator cuff tears, Rheumatol. Int., 2005, 25(6), 436-441.

11 M. Whittaker, et al., Design and Therapeutic Application of Matrix Metalloproteinase Inhibitors, Chem. Rev., 1999, 99(9), 2735-2776.

12 J. Posnett and P. J. Franks, The burden of chronic wounds in the UK, Nurs. Times, 2008, 104(3), 44-45.

13 Y. Yoshihara, et al., Biochemical markers in the synovial fluid of glenohumeral joints from patients with rotator cuff tear, J. Orthop. Res., 2001, 19(4), 573-579.

14 C. C. Dodson, et al., The long-term outcome of recurrent defects after rotator cuff repair, Am. J. Sports Med., 2010, 38(1), 35-39.

15 N. S. Nagra, et al., Mechanical properties of all-suture anchors for rotator cuff repair, Bone Jt. Res., 2017, 6(2), 82-89.

16 A. B. Wysocki, L. Staiano-Coico and F. Grinnell, Wound Fluid from Chronic Leg Ulcers Contains Elevated Levels of Metalloproteinases MMP-2 and MMP-9, J. Invest. Dermatol., 1993, 101(1), 64-68.

17 D. R. Yager, et al., Wound Fluids from Human Pressure Ulcers Contain Elevated Matrix Metalloproteinase Levels and Activity Compared to Surgical Wound Fluids, J. Invest. Dermatol., 1996, 107(5), 743-748. 
18 M. Muller, et al., Matrix metalloproteinases and diabetic foot ulcers: the ratio of MMP-1 to TIMP-1 is a predictor of wound healing, Diabetic Med., 2008, 25(4), 419-426.

19 E. A. Rayment, Z. Upton and G. K. Shooter, Increased matrix metalloproteinase-9 (MMP-9) activity observed in chronic wound fluid is related to the clinical severity of the ulcer, Br. J. Dermatol., 2008, 158(5), 951-961.

20 D. Gibson, et al., MMPs made easy, Int. Wound J., 2009, 1(1), 1-6.

21 F. Song, et al., Matrix metalloproteinase dependent and independent collagen degradation, Front. Biosci., 2006, 11, 3100-3120.

22 R. Salsas-Escat, P. S. Nerenberg and C. M. Stultz, Cleavage Site Specificity and Conformational Selection in Type I Collagen Degradation, Biochemistry, 2010, 49(19), 4147-4158.

23 H. E. Van Wart and H. Birkedal-Hansen, The cysteine switch: a principle of regulation of metalloproteinase activity with potential applicability to the entire matrix metalloproteinase gene family, Proc. Natl. Acad. Sci. U. S. A., 1990, 87(14), 5578-5582.

24 M. Gooyit, et al., A Chemical Biological Strategy to Facilitate Diabetic Wound Healing, ACS Chem. Biol., 2014, 9(1), 105-110.

25 B. P. Purcell, et al., Injectable and bioresponsive hydrogels for on-demand matrix metalloproteinase inhibition, Nat. Mater., 2014, 13(6), 653-661.

26 G. A. Skarja, et al., The effect of a hydroxamic acidcontaining polymer on active matrix metalloproteinases, Biomaterials, 2009, 30(10), 1890-1897.

27 Y.-A. Lin, et al., Rational Design of MMP Degradable PeptideBased Supramolecular Filaments, Biomacromolecules, 2014, 15(4), 1419-1427.

28 D. Brett, A Review of Collagen and Collagen-based Wound Dressings, Wounds, 2008, 20(12), 347-356.

29 J. F. Fisher and S. Mobashery, Recent advances in MMP inhibitor design, Cancer Metastasis Rev., 2006, 25(1), 115-136.

30 M. Sefton, M. May and G. Skarja, Hydroxyamate-containing materials for the inhibition of matrix metalloproteinases, Google Pat., 2004.

31 F. Renò, V. Traina and M. Cannas, Adsorption of matrix metalloproteinases onto biomedical polymers: a new aspect in biological acceptance, J. Biomater. Sci., Polym. Ed., 2008, 19(1), 19-29.

32 S. Eming, et al., The inhibition of matrix metalloproteinase activity in chronic wounds by a polyacrylate superabsorber, Biomaterials, 2008, 29(19), 2932-2940.

$33 \mathrm{~J}$. Hart, et al., The role of oxidised regenerated cellulose/ collagen in wound repair: effects in vitro on fibroblast biology and in vivo in a model of compromised healing, Int. J. Biochem. Cell Biol., 2002, 34(12), 1557-1570.

$34 \mathrm{X}$. Qiao, et al., Compositional and in Vitro Evaluation of Nonwoven Type I Collagen/Poly-dl-lactic Acid Scaffolds for Bone Regeneration, J. Funct. Biomater., 2015, 6(3), 667.

35 B. F. Pierce, et al., Photocrosslinked Co-Networks from Glycidylmethacrylated Gelatin and Poly(ethylene glycol) Methacrylates, Macromol. Biosci., 2012, 12(4), 484-493.
36 G. Tronci, S. J. Russell and D. J. Wood, Photo-active collagen systems with controlled triple helix architecture, J. Mater. Chem. B, 2013, 1(30), 3705-3715.

37 S. Ouasti, et al., Network connectivity, mechanical properties and cell adhesion for hyaluronic acid/PEG hydrogels, Biomaterials, 2011, 32(27), 6456-6470.

38 G. Tronci, et al., Protease-sensitive atelocollagen hydrogels promote healing in a diabetic wound model, J. Mater. Chem. $B, 2016,4(45), 7249-7258$.

39 R. Holmes, et al., Thiol-Ene Photo-Click Collagen-PEG Hydrogels: Impact of Water-Soluble Photoinitiators on Cell Viability, Gelation Kinetics and Rheological Properties, Polymers, 2017, 9(6), 226.

40 G. Tronci, et al., Multi-scale mechanical characterization of highly swollen photo-activated collagen hydrogels, J. R. Soc., Interface, 2015, 12, 102.

41 G. Tronci, et al., Influence of 4-vinylbenzylation on the rheological and swelling properties of photo-activated collagen hydrogels, MRS Adv., 2015, 1(8), 533-538.

$42 \mathrm{H}$. Mohammadi, et al., Inelastic behaviour of collagen networks in cell-matrix interactions and mechanosensation, J. R. Soc., Interface, 2015, 12, 102.

43 I. Van Nieuwenhove, et al., Protein functionalization revised: N-tert-butoxycarbonylation as an elegant tool to circumvent protein crosslinking, Macromol. Rapid Commun., 2014, 35(15), 1351-1355.

44 N. Rajan, et al., Preparation of ready-to-use, storable and reconstituted type I collagen from rat tail tendon for tissue engineering applications, Nat. Protoc., 2007, 1(6), 2753-2758.

45 E. M. Brown, H. M. Farrell, Jr. and R. J. Wildermuth, Influence of neutral salts on the hydrothermal stability of acid-soluble collagen, J. Protein Chem., 2000, 19(2), 85-92.

46 J. Mayne and J. J. Robinson, Comparative analysis of the structure and thermal stability of sea urchin peristome and rat tail tendon collagen, J. Cell. Biochem., 2002, 84(3), 567-574.

47 R. Jin, et al., Injectable chitosan-based hydrogels for cartilage tissue engineering, Biomaterials, 2009, 30(13), 2544-2551.

48 T. Nishio and R. Hayashi, Regeneration of a Collagen-like Circular Dichroism Spectrum from Industrial Gelatin, Agric. Biol. Chem., 1985, 49(6), 1675-1682.

49 W. T. Brinkman, et al., Photo-Cross-Linking of Type I Collagen Gels in the Presence of Smooth Muscle Cells: Mechanical Properties, Cell Viability, and Function, Biomacromolecules, 2003, 4(4), 890-895.

50 I. D. Gaudet and D. I. Shreiber, Characterization of Methacrylated Type-I Collagen as a Dynamic, Photoactive Hydrogel, Biointerphases, 2012, 7(1), 25.

51 G. Tronci, et al., Triple-helical collagen hydrogels via covalent aromatic functionalization with 1,3-phenylenediacetic acid, J. Mater. Chem. B, 2013, 1(40), 5478-5488.

52 E.-R. Kenawy, et al., A New Degradable Hydroxamate Linkage for $\mathrm{pH}$-Controlled Drug Delivery, Biomacromolecules, 2007, 8(1), 196-201.

53 F. Everaerts, et al., Quantification of carboxyl groups in carbodiimide cross-linked collagen sponges, J. Biomed. Mater. Res., Part A, 2007, 83A(4), 1176-1183. 
54 E. M. Brown, H. M. Farrell and R. J. Wildermuth, Influence of Neutral Salts on the Hydrothermal Stability of AcidSoluble Collagen, J. Protein Chem., 2000, 19(2), 85-92.

55 I. Van Nieuwenhove, et al., Protein Functionalization Revised: N-tert-butoxycarbonylation as an Elegant Tool to Circumvent Protein Crosslinking, Macromol. Rapid Commun., 2014, 35(15), 1351-1355.

56 J. V. Staros, R. W. Wright and D. M. Swingle, Enhancement by N-hydroxysulfosuccinimide of water-soluble carbodiimidemediated coupling reactions, Anal. Biochem., 1986, 156(1), 220-222.

57 S. A. Sell, et al., Cross-linking methods of electrospun fibrinogen scaffolds for tissue engineering applications, Biomed. Mater., 2008, 3(4), 045001.

$58 \mathrm{~J} . \mathrm{Hu}$, et al., Chemical cleavage of fusion proteins for highlevel production of transmembrane peptides and protein domains containing conserved methionines, Biochim. Biophys. Acta, Biomembr., 2008, 1778(4), 1060-1066.

59 D. L. Crimmins, S. M. Mische and N. D. Denslow, Chemical Cleavage of Proteins in Solution, in Current Protocols in Protein Science, John Wiley \& Sons, Inc., 2001.

60 C. J. M. Saris, et al., Hydroxylamine cleavage of proteins in polyacrylamide gels, Anal. Biochem., 1983, 132(1), 54-67.

61 S. K. Holmgren, et al., Code for collagen's stability deciphered, Nature, 1998, 392(6677), 666-667.

62 J. L. S. Lopes, et al., Distinct circular dichroism spectroscopic signatures of polyproline II and unordered secondary structures: applications in secondary structure analyses, Protein Sci., 2014, 23(12), 1765-1772.

63 K. E. Drzewiecki, et al., Circular Dichroism Spectroscopy of Collagen Fibrillogenesis: A New Use for an Old Technique, Biophys. J., 2016, 111(11), 2377-2386.

64 C.-C. Yang, et al., Matrix Metalloproteases and Tissue Inhibitors of Metalloproteinases in Medial Plica and Pannus-like Tissue Contribute to Knee Osteoarthritis Progression, PLoS One, 2013, 8(11), e79662.
65 M. S. Ågren, et al., Tumor necrosis factor- $\alpha$-accelerated degradation of type I collagen in human skin is associated with elevated matrix metalloproteinase (MMP)-1 and MMP3 ex vivo, Eur. J. Cell Biol., 2015, 94(1), 12-21.

66 L. Negron, S. Lun and B. C. H. May, Ovine forestomach matrix biomaterial is a broad spectrum inhibitor of matrix metalloproteinases and neutrophil elastase, Int. Wound J., 2014, 11(4), 392-397.

67 G. Bohn, et al., Ovine-Based Collagen Matrix Dressing: NextGeneration Collagen Dressing for Wound Care, Adv. Wound Care, 2016, 5(1), 1-10.

68 S. Chattopadhyay and R. T. Raines, Review collagen-based biomaterials for wound healing, Biopolymers, 2014, 101(8), 821-833.

69 D. Brett, A Review of Collagen and Collagen-based Wound Dressings, 2015, vol. 20, pp. 347-356.

70 S. A. Castleberry, et al., Self-Assembled Wound Dressings Silence MMP-9 and Improve Diabetic Wound Healing In Vivo, Adv. Mater., 2016, 28, 1809-1817.

71 M. Z. Končić, et al., Antiradical, Chelating and Antioxidant Activities of Hydroxamic Acids and Hydroxyureas, Molecules, 2011, 16(8), 6232.

72 W. Przychodzen, et al., Cytotoxic and Antioxidant Activities of Benzohydroxamic Acid Analogues, Bull. Korean Chem. Soc., 2013, 34, 3098-3100.

73 M. Librizzi, et al., The Histone Deacetylase Inhibitor JAHA Down-Regulates pERK and Global DNA Methylation in MDA-MB231 Breast Cancer Cells, Materials, 2015, 8(10), 7041-7047.

74 Y. Nagaoka, et al., Synthesis and cancer antiproliferative activity of new histone deacetylase inhibitors: hydrophilic hydroxamates and 2-aminobenzamide-containing derivatives, Eur. J. Med. Chem., 2006, 41(6), 697-708.

75 P. Angibaud, et al., Discovery of pyrimidyl-5-hydroxamic acids as new potent histone deacetylase inhibitors, Eur. J. Med. Chem., 2005, 40(6), 597-606. 